\title{
Artificial intelligence in drug discovery: what is new, and what is next?
}

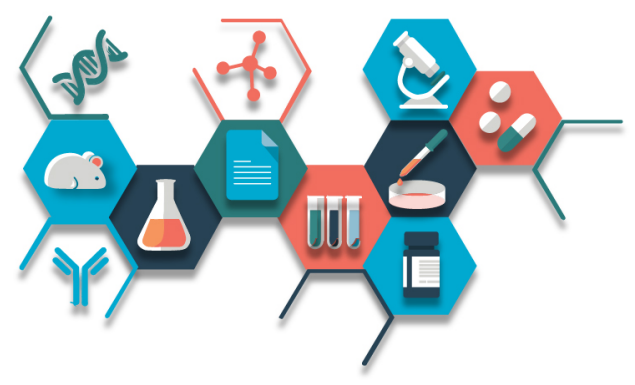

\author{
Francesca Lake*,1 (iD) \\ ${ }^{1}$ Newlands Press, Unitec House, 2 Albert Place, London, UK \\ *Author for correspondence: f.lake@future-science.com
}

\section{"While data privacy and IP are important considerations, some teams are providing machine-learning software open source."}

First draft submitted: 29 July 2019; Accepted for publication: 29 July 2019; Published online: 14 October 2019

Keywords: artificial intelligence $\bullet$ drug discovery $\bullet$ drug design $\bullet$ computational chemistry

Artificial intelligence (AI) - that is, machine intelligence - has the potential to make waves in drug discovery. However, it is not without its challenges. In this issue of Future Drug Discovery we focus on AI for drug discovery purposes, and in this editorial we take a look at how $\mathrm{AI}$ is being utilized throughout the drug design and development processes, and the positives and negatives posed by the technology.

\section{Designing a computational computer chemist}

Beginning with the earliest stages of drug discovery, AI has been harnessed to develop completely new lead compounds that exhibit desired activity in silico [1]. Combining computational de novo design with AI could allow a 'computer chemist' to learn from known useful compounds and enable the production of chemically correct and synthesizable structures with a planned biological activity. Until a study by Merk et al. at the Swiss Federal Institute of Technology (ETH; Zurich, Switzerland), AI was stymied by the huge number of possibilities involved and the potential for multiple targets [1].

"Advanced machine learning requires large well-annotated datasets that need to be compiled or generated," explained Gisbert Schneider, group leader for the study. "Also, the chemical structure of a drug alone rarely accounts for the observed pharmacological effect in a simple fashion. Most drugs have multiple biological targets and activities, and their relative importance is highly dependent on the individual genetic profile of patients, and a range of other factors. In certain areas of drug design we are confronted with inherently ill-posed problems owing to a multitude of often unknown contributing factors" [2].

As a result, previous efforts have only been able to use the approach retrospectively; however, Schneider's group was able to apply it prospectively, creating a nonhuman 'drug designer'.

"Modern machine-learning methods are very fast and can consider several design goals in parallel," he noted. "So, our drug design software was trained to recognize important features and characteristics of known drugs. The obtained models were then used to automatically assemble new molecules with these learned desired properties from scratch" [2].

\section{The computer brain versus the human brain for drug design}

$\mathrm{AI}$ is also being harnessed at the lab of Ola Engkvist, section head of the hit discovery department at AstraZeneca's Discovery Sciences Department (Cambridge, UK). In collaboration with the University of Muenster (Germany), his group has been seeking to solve the problem that drug design algorithms are not able to efficiently search the whole chemical space. Their recurrent neural networks (RNNs) are now able to, through learning.

"RNNs don't really understand chemical structures; they learn rules about how to generate novel character strings that correspond to molecules within the chemical space," he explained. However, this approach still requires human input. "Since humans decide on which chemical space to train the RNN, humans are essential to the process. What the RNN can do is generate many more molecules that are drug-like and can combine these with information 
about a drug target to home in on a certain part of the drug-like chemical space that the human may not have thought of," he continued [3].

Another neural network-based technology is Atomwise (CA, USA). Invented by Abraham Heifets and Izhar Wallach, Atomwise uses the same technology underlying 2D image and speech recognition, applying it to molecular recognition (aka, 3D image recognition). This matchmaker technology uses machine learning to screen compounds quickly; however, again it requires a human counterpart.

Heiferts noted: "To support the subtle statistical approaches, you need massive datasets. The last time I checked the NIH's PubChem database, I think there were 240 million chemical compounds in there. But that didn't exist 20-30 years ago. If you have small datasets and powerful statistics, you are going to memorize the dataset and you're not actually going to generalize, so you have to match the power of the algorithm to the size and complexity of the data. Of course, not all data is good data. $98 \%$ of those data sets don't pass our quality control filters. The old tenet of garbage in, garbage out holds - if you don't carefully go through your data, you're going to learn artefacts, you're going to fool yourself. That's part of the reason why we have a team of medicinal chemists and structural biologists, as well as machine learning and computer scientists" [4].

\section{Harnessing Al for hit identification}

One project in which Atomwise is involved is being led by Grant Wishart, leader of the Charles River ComputerAided Drug Design \& Structural Biology group (Cambridge, UK). The project is allowing Charles River, a contract research organization, to predict protein target binding of huge numbers of compounds, including synthesis ondemand libraries.

"Access to such large regions of chemical space becomes very important for those protein targets that are traditionally considered as challenging for hit finding and highly competitive 'hot targets' where access to novel chemical space is highly desired," highlighted Wishart [5].

However, he also noted that Charles River is in the early stages of applying AI for hit identification, and that it is currently too early to say it has been a success. "However, it is anticipated that the application of AI technologies in our organization will grow significantly in the near future and will have a major positive impact upon our ability to find hits for challenging targets," he continued. "This is expected to result in quicker timelines to transition projects into hit-to-lead and lead optimization phases" [5].

Again, the amount of data required for such projects has raised a challenge - both in terms of standardization, and in intellectual property (IP). "As a services company, this data is most often owned by our partners. Therefore, within individual partner drug discovery projects this data is being leveraged to advance the project and at a more holistic level we are working with our partners to seek permission, where appropriate to explore global modelling efforts," Wishart noted [5].

\section{Who holds the IP in Al drug discovery?}

The drug discovery field has always been protective of IP. Given the huge amount of data required for machine learning to be successful, it is unsurprising that issues with IP are even bigger here.

Takeshi S Komatani, of the law firm Shusaku Yamamoto (Japan), notes that IP in AI has two main aspects - subjective and objective. The subjective issues center around who the inventor is. "According to the literal interpretation of the Japanese Patent Act, an invention created solely by an AI cannot be protected by the patent act and rather goes to the public domain," he points out. This is more or less the same in the USA. "Any 'inventions' 'invented' by 'AI' would belong to the public domain. However, this needs extensive arguments and needs to be debated in detail from the respective field of professionals and expertise," he continued. For the objective side, the problem lies in what is patentable subject matter. "Practitioners are struggling to prepare a reasonable and robust specification/description to secure patent rights to AI-related technologies. With respect to copyrightable works such as those related to experimental data or the like, things are more complicated. This is because copyright does not require registration. Therefore, no one can actually identify who owns what" [6].

Another problem is the data itself. In this vein, the recent G20 summit in Osaka proposed the launch of the 'Osaka track' framework for free cross-border data flow, promoting cross-border data flow with better protection of personal information, IP and cybersecurity [7]. That meeting saw 24 countries sign the statement. It will be interesting to see how this framework affects data IP in AI-related drug discovery. 


\section{The inevitable question of ethics}

In addition to IP, AI raises various other ethical considerations. For example, patient data are invaluable to drug discovery research, but patient privacy needs to be maintained. While this is legally a requirement in many areas and multiple methods exist to ensure privacy, can it be guaranteed that data are $100 \%$ secure? "I don't think data is ever 100\% secure," commented John Mitchell (University of St Andrews, UK). "It's well understood that a determined and resourceful adversary might try to reconstruct anonymized data with reference to other available fragments of information. But medical data will be safer than most of the trails of digital footprints we leave in the sand of the internet" [8].

He believes using AI for personalized medicine might be a more pressing issue. "AI-driven medicine may well be personalized medicine, and dependent on each individual's genetic code. That means that in order to benefit, our genomes will have to be out there in some sense. If my genome is out there, what if it contains potentially bad news, such as a likelihood of developing a debilitating disease in the future? Perhaps I want to know and want my doctors to know, in order to find the optimal preventative measures and, if necessary, treatments. Maybe I'd rather not know, in order not to waste the present worrying about something that may or may not happen in the future. Should insurers have access to such information? Or what about family members? These questions are likely to become more pressing in the coming years" [8].

However, he is concerned that job attrition is the most pressing concern. Even though the Industrial Revolution was overall a good thing, many people suffered from losing their jobs to machines, and AI has the potential to repeat that process. 'The challenge is to manage an inevitable process of change and transformation while minimizing negative consequences for people and their communities. May be AI and robots will make society wealthy enough that we can afford to pay a universal citizen's income at a decent level with extra income available for those who take on paid jobs; this still provides the challenges of distributing work, opportunity and leisure time fairly while giving people something worth living for. It is also possible that we will just create more jobs for humans, probably roles as unimaginable now as a web designer or social media engagement coordinator would have been in the $1960 \mathrm{~s}^{\prime}[8]$.

\section{Open source}

While data privacy and IP are important considerations, some teams are providing machine-learning software open source. One AI effort has seen Anne Carpenter (Broad Institute of Harvard and MIT, MA, USA) creating a code to solve the bottleneck she encountered when processing cell images and making that code open source. 'CellProfiler' automatically identifies cells in images and measures properties. The later 'CellProfile Analyst' uses machine learning to recognize cells with a particular phenotype.

"This ability to quantitatively match cells based on their image-based profile is deceptively simple but there are so many applications in drug discovery," explained Carpenter. "For example, we can take cells from patients with and without a disease and compare their morphology. If we find a difference in their profiles, this can serve as a diagnostic tool, but even better we can now test thousands of drugs to find any that are able to reverse the disease profile and make cells look healthy again" [9].

Other open-source, deep-learning tools have also recently been announced, such as PaccMann, INtERAcT and PIMKL from IBM Research - Zurich (Switzerland) [10]. PaccMann incorporates transcriptomics, cellular protein interactions and compound molecular structure to predict cancer cell drug sensitivity [11]. INtERAcT uses unsupervised machine learning to examine cancer research publications and extract interactions, such as proteinprotein interactions. PIMKL is a machine-learning algorithm able to predict phenotype from multiomic data. With these methods only just openly available, it will be interesting to see how they are utilized going forward.

\section{So what is next for Al in drug discovery?}

Clearly, AI is already helping drug discovery - it can help identify drug targets, find good molecules from data libraries, suggest chemical modifications, identify candidates for repurposing and so on. However, in the short term it has a number of challenges to overcome. The buzz around AI could be its own downfall - Mitchell noted: "often new approaches to drug discovery get overhyped, like combinatorial chemistry did a few years ago, and failure to manage unrealistic expectations leads to an inevitable let down and to a perceived bursting of the bubble. It's better to expect incremental advances and perhaps to be pleasantly surprised than to promise a revolution that never materializes" [8]. With that, AI needs to prove itself. Heifets agrees that this is a huge challenge: "It's insufficient to predict yesterday's weather. You have to predict tomorrow's weather, and get it right over and over and over again. I think in this field, whether it is AI or anything else, you have to be able to show a couple dozen successes when nobody knew what the answer was" [4]. 
The availability of robust datasets and the need for investment to access AI technology could also prove to be hurdles.

However, there is plenty of excitement over the future; "I think the next 5-10 years will be really exciting," enthused Engkvist. "I think one of the next big advances will be a much tighter integration with automation that allows us to move from an augmented drug design paradigm where the design chemist takes all the decisions to an autonomous drug design paradigm, where the system can autonomously decide which compound to make next" [3]. Schneider agrees autonomy is the next exciting step: "I expect fully autonomous laboratories iterate through the design-make-test-analyze cycle of drug discovery without direct human intervention. The result could be the delivery of better starting points for drug discovery faster" [2].

\section{Financial \& competing interests disclosure}

The authors have no relevant affiliations or financial involvement with any organization or entity with a financial interest in or financial conflict with the subject matter or materials discussed in the manuscript. This includes employment, consultancies, honoraria, stock ownership or options, expert testimony, grants or patents received or pending, or royalties.

No writing assistance was utilized in the production of this manuscript.

Open access

This work is licensed under the Attribution-NonCommercial-NoDerivatives 4.0 Unported License. To view a copy of this license, visit http://creativecommons.org/licenses/by-nc-nd/4.0/

\section{Disclaimer}

This article was written as part of a collaboration with Eureka, the Charles River Labs blog. The interviews included form part of an interview series available on Eureka. The opinions expressed in this Editorial are those of the interviewees and do not necessarily reflect the views of Newlands Press Ltd.

\section{References}

1. Merk D, Friedrich L, Grisoni F, Schneider G. De novo design of bioactive small molecules by artificial intelligence (2018). https://onlinelibrary.wiley.com/doi/full/10.1002/minf.201700153

2. Eureka. A chemistry-savvy AI joins the lab team (2019). https://eureka.criver.com/a-chemistry-savvy-ai-joins-the-lab-team/

3. Eureka. The computer as drug hunter (2019). https://eureka.criver.com/the-computer-as-drug-hunter/

4. Kelder R. Commercializing deep neural networks for drug discovery (2019). https://eureka.criver.com/commercializing-deep-neural-networks-for-drug-discovery/

5. Eureka. The future of drug discovery: AI impacting upon hit ID strategies (2019). https://eureka.criver.com/the-future-of-drug-discovery-ai-impacting-upon-hit-id-strategies/

6. Eureka. For AI-enabled drug discoveries, who owns the science? (2019). https://eureka.criver.com/for-ai-enabled-drug-discoveries-who-owns-the-science/

7. Sugiyama S. Abe heralds launch of 'Osaka Track' framework for free cross-border data flow at G20. The Japan Times, 28th June (2019). https://www.japantimes.co.jp/news/2019/06/28/national/abe-heralds-launch-osaka-track-framework-f ree-cross-border-data-flow-g20/ \#.XTXXFuhKijR

8. Eureka. Ethical dilemmas that artificial intelligence raise in the lab (2019). https://eureka.criver.com/ethical-dilemmas-that-artificial-intelligence-raise-in-the-lab/

9. Eureka. Image-based cell profiling (2019). https://eureka.criver.com/image-based-cell-profiling/

10. Manica M, Cadow J. Novel AI tools to accelerate cancer research (2019). https://www.ibm.com/blogs/research/2019/07/ai-tools-for-cancer-research/

11. Oskooei A, Born J, Manica M, Subramanian V, Sáez-Rodríguez J, Martínez MR. PaccMann: prediction of anticancer compound sensitivity with multi-modal attention-based neural networks (2019). https://arxiv.org/abs/1811.06802 